\title{
ON CERTAIN INTEGRABLE NONLINEAR MEMBRANE SOLUTIONS*
}

\author{
$B Y$ \\ CHIEN-HENG WU \\ University of Illinois, Chicago
}

1. Introduction. We study the class of axially symmetrical deformations of an elastic sheet characterized by a strain energy function satisfying certain inequalities pertinent to finite elasticity. It is found that the system of nonlinear equations can be reduced to quadratures if either the undeformed or the deformed middle surface is cylindrical.

When the undeformed state is a cylindrical surface, the equation of equilibrium for the direction tangent to the meridian curve has a first integral if the strain energy function does not depend on position explicitly. This fact has been discovered independently by Pipkin [1] and $\mathrm{Wu}$ [2]. While a specific problem was solved in [2], the deduction given in [1] was completely general. The fact that the equation of equilibrium for the direction normal to the middle surface has a first integral is known [3]. We use these two integrals to obtain the general solution of our problem.

When the deformed state is a cylindrical surface, the equations of equilibrium reduce to very simple forms which can be integrated even if the material is meridianly inhomogeneous.

2. Formulation. We consider the class of axially symmetrical problems in which the middle surface of an elastic sheet forms a surface of revolution before and after deformation. Let the middle surface $M$ of the undeformed sheet be characterized by a meridian curve $C$ and let the middle surface $m$ of the deformed sheet be characterized by a meridian curve $c$. The thickness $H$ of the undeformed sheet is assumed to be constant.

We choose a cylindrical coordinate system $(R, \theta, Z)$, the $z$-axis of which coincides with the axis of symmetry of the sheet. We assume that any point $P(R, \theta, Z)$ in $M$ is carried by the deformation to the point $p(r, \theta, z)$ in $m$. Let $C$ have arc length $S$ and let $c$ have arc length $s$. We introduce a set of unit vectors $\left(\mathbf{a}_{1}, \mathbf{a}_{2}, \mathbf{a}_{3}\right), \mathbf{a}_{1}$ being tangent to the curve $c$ and pointing to the direction of increasing $s, a_{2}$ being tangent to the lines of azimuth and pointing to the direction of increasing $\theta$. From the symmetry of the system it follows that $\left(a_{1}, a_{2}, a_{3}\right)$ are the principal directions of strain at $P$. If we denote by $\lambda_{1}, \lambda_{2}, \lambda_{3}$ the principal extension ratios in these three directions, then

$$
\begin{aligned}
& \lambda_{1}=d s / d S, \\
& \lambda_{2}=r / R, \\
& \lambda_{3}=h / H
\end{aligned}
$$

where $h$ is the thickness of the deformed sheet.

* Received April 23, 1969. The research reported herein was supported by the Air Force Office of Scientific Research under Grant AFOSR-537-67. The paper was written while the author was a visiting member at the Courant Institute of Mathematical Sciences, New York University. 
We now assume that the elastic sheet is characterized by a strain energy function $\omega=\omega\left(\lambda_{1}, \lambda_{2}, S\right)$. It is shown in Appendix I that, for such an elastic sheet, the meridian and azimuthal stress resultants $t_{1}$ and $t_{2}$ are given by

$$
\begin{aligned}
& t_{1}=\frac{1}{\lambda_{2}} \omega_{\lambda_{2}} \\
& t_{2}=\frac{1}{\lambda_{1}} \omega_{\lambda_{2}}
\end{aligned}
$$

where the subscripts on $\omega$ denote partial differentiation with respect to the indicated argument. We require that the strain energy function $\omega$ satisfies the conditions

$\frac{1}{\lambda_{1} \lambda_{2}}\left[\omega_{\lambda_{1} \lambda_{1}} \omega_{\lambda_{2} \lambda_{2}}-\left(\omega_{\lambda_{1} \lambda_{2}}-\frac{1}{\lambda_{2}} \omega_{\lambda_{1}}\right)\left(\omega_{\lambda_{1} \lambda_{2}}-\frac{1}{\lambda_{1}} \omega_{\lambda_{2}}\right)\right] \neq 0, \quad \frac{1}{\lambda_{2}} \omega_{\lambda_{1} \lambda_{2}}>0, \frac{1}{\lambda_{1}} \omega_{\lambda_{2} \lambda_{2}}>0$.

These inequalities correspond respectively to the conditions of "invertibility of forcestretch" and "tension-extension" discussed by Truesdell and Toupin [4].

It is also shown in Appendix I that the stress resultants satisfy the equations of equilibrium

$$
\begin{gathered}
d\left(r t_{1}\right) / d s=t_{2} d r / d s . \\
t_{1}(d \phi / d s)+(\sin \phi) t_{2} / r=q
\end{gathered}
$$

where $-q \mathbf{a}_{3}$ is the externally applied force per unit deformed area and $\phi$ is the tangent angle such that

$$
\begin{aligned}
& d r / d s=\cos \phi, \\
& d z / d s=\sin \phi .
\end{aligned}
$$

Thus we have eight equations (2.1), (2.2), (2.4), (2.5), (2.7), (2.8), (2.9) and (2.10) for the eight unknowns $\lambda_{1}, \lambda_{2}, t_{1}, t_{2}, r, z, s$ and $\phi$. In general, the unknown $h$ does not enter into the analysis. If the elastic sheet is made of Mooney material (cf. Appendix I), then the thickness $h$ of the deformed sheet can be determined from the condition of incompressibility

$$
h=H \lambda_{3}=H / \lambda_{1} \lambda_{2} .
$$

Various numerical procedures are used to integrate the system of equations. In Secs. 3 and 4, we show that the system can be reduced to quadratures if either the undeformed or the deformed middle surface is cylindrical.

3. Deformation from a tube. The middle surface $M$ of the undeformed sheet is assumed to be a circular cylindrical surface and the elastic sheet is homogeneous, i.e. $\omega$ does not depend explicitly on $S$.

Let $M$ be generated by the meridian curve

$$
C: \quad R=1, Z=S, \quad 0 \leq S \leq L \leq \infty .
$$

The principal extension ratios become

$$
\begin{aligned}
& \lambda_{1}=d s / d Z, \\
& \lambda_{2}=r .
\end{aligned}
$$


The problem now is to solve the system of Eqs. (3.2), (3.3), (2.4), (2.5), (2.7), (2.8), (2.9) and (2.10).

Eq. (2.7) can be written as

$$
d\left(r t_{1}\right) / d r=t_{2}
$$

if $d r / d s$ does not vanish. Multiplying (3.4) by $\lambda_{1} d \lambda_{2} / d r\left(=\lambda_{1}\right)$ and using (2.4), (2.5), and (3.3), we obtain

$$
\begin{aligned}
\omega_{\lambda_{2}} d \lambda_{2} / d r & =\lambda_{1} d \omega_{\lambda_{1}} / d r \\
& =d\left(\lambda_{1} \omega_{\lambda_{1}}\right) / d r-\omega_{\lambda_{1}} d \lambda_{1} / d r .
\end{aligned}
$$

Hence,

$$
\omega-\lambda_{1} \omega_{\lambda_{1}}=a
$$

where $a$ is a constant of integration. This deduction was given by Pipkin [1].

If the elastic sheet is made of isotropic incompressible Mooney material (cf. Appendix I), then (3.5) reduces to

$$
\lambda_{1}^{2}-3\left(r \lambda_{1}\right)^{-2}+k\left(r \lambda_{1}\right)^{2}-3 k \lambda_{1}^{-2}-r^{2}-k r^{-2}=a .
$$

This expression was obtained by $\mathrm{Wu}$ [2], using a different approach.

We consider in great detail the general solutions of the following two cases.

CASE I. Pressure $q$ is a given function of $r$.

Since $\lambda_{2}=r$, (3.5) can be solved to yield

$$
\lambda_{1}=\lambda_{1}^{*}(r, a) .
$$

This is guaranteed by the fact that the implicit function condition for (3.5) is satisfied by the assumptions (2.6) imposed on $\omega$. Then Eqs. (2.4), (2.5) yield

$$
\begin{aligned}
& t_{1}=t_{1}^{*}(r, a)=\omega_{\lambda_{1}}\left(\lambda_{1}^{*}, r\right) / r, \\
& t_{2}=t_{2}^{*}(r, a)=\omega_{\lambda_{2}}\left(\lambda_{1}^{*}, r\right) / \lambda_{1}^{*} .
\end{aligned}
$$

It is noted that for a given problem, i.e. for a specific value of $a$, the values of $\lambda_{1}, t_{1}$ and $t_{2}$ at a point $p(r, \theta, z)$ of $m$ depend only on $r$ but not on $z$.

Multiplying (2.8) by $r(d r / d s)$ and using (2.7), we obtain

$$
\begin{aligned}
r \frac{d r}{d s}\left(\frac{d \phi}{d s} t_{1}+\frac{\sin \phi}{r} t_{2}\right) & =\frac{d}{d s}(\sin \phi) r t_{1}+\sin \phi \frac{d}{d s}\left(r t_{1}\right) \\
& =\frac{d}{d s}\left(r t_{1} \sin \phi\right)=q r \frac{d r}{d s} .
\end{aligned}
$$

Integration yields

$$
r t_{1} \sin \phi=\int q(r) r d r+b
$$

where $b$ is a constant of integration. Eq. (3.9) shows that $b$ is proportional to the total (axial) force applied to a circular cross-section of the sheet. If $r t_{1} \neq 0$, then (3.9) implies

$$
\phi=\phi^{*}(r, a, b)=\operatorname{Sin}^{-1}\left[\left(\int q r d r+b\right) / r t^{*}\right] .
$$


From equation (3.2)

$$
d s / d Z=(d s / d r)(d r / d Z)=\lambda_{1}
$$

and hence

$$
d r / d Z=\lambda_{1} \cos \phi .
$$

This equation can be integrated to yield

$$
Z=Z^{*}\left(r, a, b, r_{0}\right)=\int_{r_{0}}^{r} \frac{d r_{1}}{\lambda_{1}^{*}\left(r_{1}\right) \cos \phi^{*}\left(r_{1}\right)}
$$

where $r_{0}=\left.r\right|_{z=0}$. It is also convenient to set

$$
s=0, \quad z=0 \quad \text { at } \quad Z=0 .
$$

It follows from (2.9), (2.10) and (3.14) that

$$
\begin{aligned}
& s=s^{*}\left(r, a, b, r_{0}\right)=\int_{r_{0}}^{r} \frac{d r_{1}}{\cos \phi^{*}\left(r_{1}\right)}, \\
& z=z^{*}\left(r, a, b, r_{0}\right)=\int_{r_{0}}^{r} \tan \phi^{*}\left(r_{1}\right) d r_{1} .
\end{aligned}
$$

It should be mentioned that the integrals appearing in equations (3.14), (3.15) and (3.16) are improper when $\cos \phi^{*}=0$. However, a simple calculation given in Appendix II shows that these integrals do exist.

The system of Eqs. (3.2), (3.3), (2.4), (2.5), (2.7-2.10) is of fifth order and has the general solution given by (3.6), (3.7), (3.8), (3.10), (3.13), (3.15) and (3.16). Since we have conveniently set up the coordinates in such a way that (3.14) is satisfied, the general solution contains only three constants $a, b$ and $r_{0}$. These constants must then be chosen to satisfy the set of transcendental equations corresponding to a given set of boundary conditions. The number of solutions of these transcendental equations will be considered in subsequent work.

We now consider the solution of the initial value problem with the initial conditions:

$$
s=0, \quad z=0, \quad r=r_{0}, \quad \phi=\phi_{0}, t_{1}=t_{0} \quad \text { at } Z=0 .
$$

Substituting the third and the fifth conditions of (3.17) into (2.4), we find

$$
\left.\lambda_{1}\right|_{z=0}=\lambda_{0}\left(r_{0}, t_{0}\right)
$$

since $\omega_{\lambda_{1} \lambda_{1}} /\left.\lambda_{2}\right|_{z=0} \neq 0$. Then (3.5) yields

$$
a=a_{0}\left(r_{0}, t_{0}\right)=\left.\left(\omega-\lambda_{1} \omega_{\lambda_{2}}\right)\right|_{\lambda_{2}=\lambda_{0}} ; \lambda_{2}=r_{0} .
$$

Thus, the solution of the initial value problem is

$$
\begin{aligned}
\lambda_{1} & =\lambda_{1}^{0}\left(r, r_{0}, t_{0}\right)=\lambda_{1}^{*}\left(r, a_{0}\left(r_{0}, t_{0}\right)\right), \\
t_{1} & =t_{1}^{0}\left(r, r_{0}, t_{0}\right)=\omega_{\lambda_{1}}\left(\lambda_{1}^{0}, r\right) / r, \\
t_{2} & =t_{2}^{0}\left(r, r_{0}, t_{0}\right)=\omega_{\lambda_{2}}\left(\lambda_{1}^{0}, r\right) / \lambda_{1}^{0}, \\
\phi & =\phi^{0}\left(r, r_{0}, t_{0}, \phi_{0}\right)=\sin ^{-1}\left(\int_{r_{0}}^{r} q\left(r_{1}\right) r_{1} d r_{1}+r_{0} t_{0} \sin \phi_{0}\right) / r t_{1}^{0},
\end{aligned}
$$




$$
\begin{aligned}
& Z=Z^{0}\left(r, r_{0}, t_{0}, \phi_{0}\right)=\int_{r_{0}}^{r} \frac{d r_{1}}{\lambda_{1}^{0}\left(r_{1}\right) \cos \phi^{0}\left(r_{1}\right)}, \\
& s=s^{0}\left(r, r_{0}, t_{0}, \phi_{0}\right)=\int_{r_{0}}^{r} \frac{d r_{1}}{\cos \phi^{0}\left(r_{1}\right)}, \\
& z=z^{0}\left(r, r_{0}, t_{0}, \phi_{0}\right)=\int_{r_{0}}^{r} \tan \phi^{0}\left(r_{1}\right) d r_{1} .
\end{aligned}
$$

The problem of a circular cylindrical sheet stretched in the direction of its axis by pulling the end circles apart while maintianing them undeformed has been considered by Stoker [5]. The equations were solved numerically by a finite difference method. This step can be eliminated by using the present results. To illustrate the procedure, let the radius of the cylindrical sheet be unity and let the length be $2 L$. The sheet is stretched to a length $2 l>2 L$. We choose a cylindrical coordinate system $(R, \theta, Z)$, the $Z$-axis of which coincides with the axis of symmetry of the cylindrical sheet; the plane $Z=0$ contains one of the end circles. Because of the symmetry of the deformation, we consider only one half of the sheet, i.e. $0 \leq Z \leq L$.

The problem now is to solve the system of Eqs. (3.2), (3.3), (2.4), (2.5), (2.7-2.10) with $q=0$ subject to the boundary conditions:

$$
\begin{array}{llll}
s=0, & z=0, & r=1 & \text { at } \\
z=l, & \phi=\pi / 2 & \text { at } & Z=L
\end{array}
$$

The solution is given by $f^{*}\left(r, a, b, r_{0}\right)$ where $f$ is a generic symbol and the constants $a, b, r_{0}$ are the solutions of the transcendental equations:

$$
\begin{aligned}
r_{0} & =1, \\
Z^{*}\left(r_{1}, a, b, r_{0}\right) & =L, \\
\phi^{*}\left(r_{1}, a, b\right) & =\pi / 2, \\
z^{*}\left(r_{1}, a, b, r_{0}\right) & =l .
\end{aligned}
$$

In the above equations $r_{1}=\left.r\right|_{z=L}$ is the deformed radius at the "throat".

Alternatively, we may wish to consider the initial value problem with the initial conditions:

$$
s=0, z=0, \quad r=1, \quad t=t_{0}, \phi=\phi_{0} \quad \text { at } \quad Z=0 .
$$

The solution is given by $f^{0}\left(r, r_{0}, t_{0}, \phi_{0}\right)$ where $f$ is again a generic symbol and $r_{0}=1$. The constants $t_{0}$ and $\phi_{0}$ have to be adjusted in such a way that both conditions (3.27) at $Z=L$ are satisfied.

CAsE II. The middle surface $m$ of the deformed sheet is given.

Let $m$ be generated by a meridian curve $c$ which is a portion of a given curve parametrized by

$$
r=\rho(\sigma), \quad z=\zeta(\sigma), \quad \zeta(0)=0
$$

where $\rho$ and $\zeta$ are continuous functions of the arc length $\sigma$. The functions $\rho$ and $\zeta$ may have discontinuous derivatives.

The undeformed circular cylindrical sheet (3.1) is applied onto the surface of revolu- 
tion generated by (3.29). We wish to determine all the physical quantities as functions of $Z$. In particular, we wish to determine the pressure $q$ between the sheet and the given surface. While it is difficult to impose physically reasonable, a priori restrictions on $\rho$ and $\zeta$, we assume that they do not give rise to negative pressure.

Since the deformed middle surface is known, the equations which remain to be solved are (3.2), (3.3), (2.4), (2.5), (2.7), (2.8) and

$$
d s / d \sigma=1 .
$$

Moreover, Eq. (2.8) decouples from the other equations, and is simply an algebraic equation for the determination of the pressure $q$.

The system of equations also admits the first integral (3.5). Since $\lambda_{2}=r=\rho(\sigma)$, and because of (2.6), Eq. (3.5) can be solved to yield

The stress resultants are

$$
\lambda_{1}=\lambda_{1}^{+}(\sigma, a) .
$$

$$
\begin{aligned}
& t_{1}=t_{1}^{+}(\sigma, a)=\omega_{\lambda_{1}}\left(\lambda_{1}^{+}, \rho(\sigma)\right) / \rho(\sigma), \\
& t_{2}=t_{2}^{+}(\sigma, a)=\omega_{\lambda_{2}}\left(\lambda_{1}^{+}, \rho(\sigma)\right) / \lambda_{1}^{+} .
\end{aligned}
$$

Eqs. (3.2), (3.30) and (3.31) yield

$$
Z=Z^{+}\left(\sigma, a, \sigma_{0}\right)=\int_{\sigma_{0}}^{\sigma} \frac{d \sigma_{1}}{\lambda_{1}^{+}\left(\sigma_{1}\right)}
$$

where $\sigma_{0}=\left.\sigma\right|_{z=0}$.

Thus the general solution of this case is given by (3.31)-(3.34) which involve two constants of integration $a$ and $\sigma_{0}$.

We also obtain explicit expression for the solution of the initial value problem with the initial conditions:

$$
\sigma=\sigma_{0}\left(r=\rho\left(\sigma_{0}\right)\right) \text { and } t_{1}=t_{0} \text { at } Z=0 .
$$

These are the same conditions used to derive (3.18). Thus

$$
\begin{aligned}
\lambda_{1} & =\lambda_{1}^{\Delta}\left(\sigma, \sigma_{0}, t_{0}\right)=\lambda_{1}^{0}\left(\rho(\sigma), \rho\left(\sigma_{0}\right), t_{0}\right), \\
t_{1} & =t_{1}^{\Delta}\left(\sigma, \sigma_{0}, t_{0}\right)=\omega_{\lambda_{1}}\left(\lambda_{1}^{\Delta}, r\right) / \rho(\sigma), \\
t_{2} & =t_{2}^{\Delta}\left(\sigma, \sigma_{0}, t_{0}\right)=\omega_{\lambda_{2}}\left(\lambda_{1}^{\Delta}, r\right) / \lambda_{1}^{\Delta} .
\end{aligned}
$$

Finally, (3.2), (3.30) and (3.31) yield

$$
Z=Z^{\Delta}\left(\sigma, \sigma_{0}, t_{0}\right)=\int_{\sigma_{0}}^{\sigma} \frac{d \sigma_{1}}{\lambda_{1}^{\Delta}\left(\sigma_{1}\right)} .
$$

Eqs. (3.36-3.39) constitute the complete solution for the initial value problem. The special case of a Mooney tube stretched into an annulus has been given explicitly in [2].

We conclude this section by remarking that if the curve (3.29) has discontinuous first derivative at $\sigma=\sigma_{d}$, then a line distribution of pressure will result there. The intensity of the line load is

$$
2 t_{1}^{+}\left(\sigma_{d}, a\right) \sin \frac{1}{2}\left(\phi\left(\sigma_{d}+\right)-\phi\left(\sigma_{d}-\right)\right)
$$

and the line of action bisects the angle formed by (3.25) at $\sigma=\sigma_{d}$. 
4. Deformation to a tube. We consider the deformation of an inhomogeneous elastic sheet ${ }^{1}$ of revolution applied onto a circular cylindrical surface. Let the meridian curves of the middle surfaces $M$ and $m$ have the parametric representations:

$$
\begin{aligned}
& C: R=R_{0}(S), \quad Z=Z_{0}(S), \quad R_{0} \leq 1, \quad Z_{0}(0)=0, \quad 0 \leq S \leq L<\infty \\
& c: r=1, \quad z=s, \quad 0 \leq s<\infty .
\end{aligned}
$$

The principal extension ratios now become

$$
\lambda_{1}=d z / d S, \quad \lambda_{2}=1 / R=1 / R_{0}(S),
$$

and the equations of equilibrium (2.7), (2.8) reduce to

$$
\begin{aligned}
d t_{1} / d z & =0, \\
q & =t_{2} .
\end{aligned}
$$

It follows from (4.4) and (2.4) that

$$
t_{1}=\frac{1}{\lambda_{2}} \frac{\partial}{\partial \lambda_{1}} \omega\left(\lambda_{1}, \lambda_{2}, S\right)=t_{0}
$$

where $t_{0}$ is a constant of integration. In view of the second condition of (2.6) and the fact that $\lambda_{2}$ is a known function of $S$, Eq. (4.6) can be inverted to yield $\lambda_{1}=\lambda_{1}\left(S, t_{0}\right)$. Thus

$$
z=\int_{0}^{S} \lambda_{1}\left(S_{1}, t_{0}\right) d S_{1} .
$$

This completes the solution. We consider the following examples:

Example 1. An elastic tube of radius $0<R<1$ made of Mooney material is applied on a rigid cylinder of radius $r=1$.

Eqs. (1.10) and (4.6) with $t_{1} \equiv 0$ imply that $\lambda_{1}=R^{1 / 2}$ and hence $z=R^{1 / 2} Z$. Thus the length of the tube never shrinks to zero. The pressure between the tube and cylinder is

$$
q=t_{2}=\left(R^{-3 / 2}-R^{3 / 2}\right)\left(1+K_{m} R\right) .
$$

Example 2. An elastic tube of radius $0<R<1$ made of classical material is applied on a rigid cylinder of radius $r=1$.

Eqs. (1.9) and (4.6) with $t_{1} \equiv 0$ imply that

$$
z=\left[\frac{2+K_{c}}{1+K_{c}}-\frac{1}{\left(1+K_{c}\right) R}\right] Z .
$$

Thus, the length of a tube of initial radius $R=1 /\left(2+K_{c}\right)$ is shrunk to zero. Similar phenomena have been found by Stoker [5] and Sensenig [6]. The pressure is

$$
q=t_{2}=K_{c}\left(2+K_{c}\right)(1-R) /\left[\left(2+K_{c}\right) R-1\right] .
$$

Example 3. An elastic annulus $C: Z=0, R=S, 0<\delta \leq S \leq 1$, made of Mooney material is applied on a rigid cylinder of radius $r=1$.

Eqs. (1.10) and (4.6) with $t_{1} \equiv 0$ imply that

$$
\lambda_{1}=d z / d R=R^{1 / 2}
$$

1The elastic sheet is characterized by a strain energy function $\omega=\omega\left(\lambda_{1}, \lambda_{2}, S\right)$. 
and hence

$$
z=\frac{2}{3}\left(R^{3 / 2}-\delta^{3 / 2}\right) .
$$

The pressure is

$$
q=t_{2}=\left(R^{-3 / 2}-R^{3 / 2}\right)\left(1+K_{m} R\right) .
$$

Acknowledgment. The author is indebted to Professor J. B. Keller who read the manuscript and offered many helpful criticisms.

\section{APPENDIX I: Derivation of Governing Equations}

Let us consider an elastic sheet which in the undeformed state is characterized by the meridian curve

$$
C: R=R(S), \quad Z=Z(S), \quad Z(0)=0, \quad 0 \leq S \leq L .
$$

The sheet is subjected to externally applied normal pressure

$$
\mathrm{q}=-q \mathrm{a}_{3}
$$

per unit deformed area. The deformed state which is characterized by the coordinates of $p(r, \theta, z)$ is assumed to satisfy the following variational expression

$$
\left.\delta \int_{0}^{L} 2 \pi R \omega\left(\lambda_{1}, \lambda_{2}, S\right) d S=-\int_{0}^{L} 2 \pi r q \lambda_{1} \delta u_{3} d S+2 \pi r \bar{t}_{1} \delta u_{1}\right]_{0}^{L} .
$$

Here $\omega$ is a strain energy function per unit undeformed area, $\vec{t}_{1}$ is the force per unit deformed arc length acting at the ends of the sheet and $\delta u_{1}, \delta u_{3}$ are respectively the $\mathbf{a}_{1}$ and $\mathbf{a}_{2}$ components of the virtual displacement about the equilibrium position $p(r, \theta, z)$. The presence of $S$ in $\omega$ allows for inhomogeneous material properties.

Let prime denote differentiation with respect to $S$. It is easy to show that

$$
\begin{aligned}
\delta \lambda_{1} & =\operatorname{Cos} \phi \delta r^{\prime}+\operatorname{Sin} \phi \delta z^{\prime} \\
& =\delta u_{1}^{\prime}-\phi^{\prime} \delta u_{3}, \\
\delta \lambda_{2}= & \frac{\delta r}{R}=\frac{\operatorname{Cos} \phi}{R} \delta u_{1}-\frac{\operatorname{Sin} \phi}{R} \delta u_{3}
\end{aligned}
$$

where $\phi$ is the tangent angle. Equations (I-3), (I-4) imply that

$$
\begin{aligned}
\int_{0}^{L}\left\{-\left[\left(R \omega_{\lambda_{1}}\right)^{\prime}-\operatorname{Cos} \phi \omega_{\lambda_{2}}\right] \delta u_{1}-\left[R \phi^{\prime} \omega_{\lambda_{2}}+\operatorname{Sin} \phi \omega_{\lambda_{2}}-r \lambda_{1} q\right] \delta u_{3}\right\} d S & \\
& \left.+\left(R \omega_{\lambda_{2}}-r \bar{t}_{1}\right) \delta u_{1}\right]_{0}^{L}=0 .
\end{aligned}
$$

Then, the necessary and sufficient conditions for (I-5) to hold are the Euler equations

$$
\begin{gathered}
d\left(r t_{1}\right) / d s=t_{2} d r / d s, \\
t_{1}(d \phi / d s)+(\operatorname{Sin} \phi) t_{2} / r=q,
\end{gathered}
$$

and the boundary conditions

$$
\left.\left(t_{1}-t_{1}\right) \delta u_{1}\right]_{0}^{L}=0
$$


where the notations

$$
t_{1}=\omega_{\lambda_{1}} / \lambda_{2}, \quad t_{2}=\omega_{\lambda_{2}} / \lambda_{1}
$$

have been used. Physically, these quantities are the stress resultants in the meridian and azimuthal directions.

Two types of strain energy function are commonly used, namely the classical function $\omega_{c}$ and the Mooney function $\omega_{m}$. The nondimensional forms of these functions and the corresponding constitutive relations are

$$
\begin{aligned}
2 \omega_{c} & =\left(\lambda_{1}+\lambda_{2}-2\right)^{2}+K_{c}\left[\left(\lambda_{1}-1\right)^{2}+\left(\lambda_{2}-1\right)^{2}\right], \quad K_{c}=2 \mu / \lambda, \\
t_{1} & =\frac{1}{\lambda_{2}}\left[\left(\lambda_{1}+\lambda_{2}-2\right)+K_{c}\left(\lambda_{1}-1\right)\right], \\
t_{2} & =\frac{1}{\lambda_{1}}\left[\left(\lambda_{1}+\lambda_{2}-2\right)+K_{c}\left(\lambda_{2}-1\right)\right], \\
2 \omega_{m} & =\left(\lambda_{1}^{2}+\lambda_{2}^{2}+\frac{1}{\lambda_{1}^{2} \lambda_{2}^{2}}\right)+K_{m}\left(\lambda_{1}^{2} \lambda_{2}^{2}+\frac{1}{\lambda_{1}^{2}}+\frac{1}{\lambda_{2}^{2}}\right), \quad K_{m}=\frac{C_{2}}{C_{1}}, \\
t_{1} & =\left(\frac{\lambda_{1}}{\lambda_{2}}-\frac{1}{\lambda_{1}^{3} \lambda_{2}^{3}}\right)\left(1+K_{m} \lambda_{2}^{2}\right), \\
t_{2} & =\left(\frac{\lambda_{2}}{\lambda_{1}}-\frac{1}{\lambda_{1}^{3} \lambda_{2}^{3}}\right)\left(1+K_{m} \lambda_{1}^{2}\right)
\end{aligned}
$$

where $\lambda, \mu$ are the Lamé constants and $C_{1}, C_{2}$ are the Mooney constants. To obtain the dimensional forms, multiply $\omega_{c}$ by $2 \lambda$ and $\omega_{m}$ by $2 C_{1} H$.

\section{APPENDIX II: Integrability of Eqs. (3.13), (3.15) and (3.16)}

It suffices to show that the integral

$$
\begin{aligned}
& I=\int_{u}^{0} \frac{d r}{\cos \phi^{*}}, \\
& \left.\cos \phi^{*}\right|_{r=u}=0, \\
& \cos \phi^{*} \neq 0 \quad \text { for } u<r \leq v
\end{aligned}
$$

exists. Equation (3.9) implies

$$
\begin{aligned}
& \sin \phi^{*}=Q / r t_{1}^{*}, \\
& Q=\int q r d r+b .
\end{aligned}
$$

Thus

$$
\sin ^{2} \phi^{*}=\frac{Q^{2}}{r^{2} t_{1}^{* 2}}=1+\left.(r-u) \frac{d}{d r}\left(\frac{Q^{2}}{r^{2} t_{1}^{* 2}}\right)\right|_{\bar{r}}
$$

where $u \leq \bar{r} \leq r$. Differentiating and applying (3.4), we have

$$
\frac{d}{d r}\left(\frac{Q^{2}}{r^{2} t_{1}^{* 2}}\right)=\frac{2 Q^{2}}{r^{2} t_{1}^{* 3} \sin \phi^{*}}\left(q-\frac{\sin \phi^{*}}{r} t_{2}^{*}\right) \neq 0
$$


for $u<r<v$ by equation (2.8). This implies that

$$
0<\left|\frac{1}{\cos \phi^{*}}\right| \leq \frac{M}{|r-u|^{1 / 2}}
$$

where

$$
\frac{1}{M I^{2}}=\operatorname{Min}_{u<r \leq v}\left|\frac{d}{d r}\left(\frac{Q^{2}}{r^{2}} \frac{t_{1}^{*^{2}}}{2}\right)\right| .
$$

Equation (II-2) implies that (II-1) is integrable.

\section{REFERENCES}

1. Pipkin, A. C., Integration of an equation in membrane theory, ZAMP 19, 818-819 (1968)

2. Wu, C. H., Tube to annulus—an exact nonlinear membrane solution, Quart. Appl. Math. 27, 489$496(1970)$

3. Green, A. E. and Adkins, J. E., Large elastic deformation, Clarendon Press, Oxford, 1960.

4. Truesdell, C. and Toupin, R., Static grounds for inequalities in finite strain of elastic materials, Arch. Rat. Mech. Anal. 12, 1-33 (1963)

5. Stoker, J. J., Topics in non-linear elasticity, Courant Institute of Mathematical Sciences, 1964.

6. Sensenig, C. B., Instability of thick elastic solids, Comm. Pure Appl. Math., 17, 451-491 (1964) 